\title{
TAILS OF STOPPED RANDOM PRODUCTS: THE FACTOID AND SOME RELATIVES
}

\author{
ANTHONY G. PAKES, ${ }^{*}$ University of Western Australia
}

\begin{abstract}
The upper tail behaviour is explored for a stopped random product $\prod_{j=1}^{N} X_{j}$, where the factors are positive and independent and identically distributed, and $N$ is the first time one of the factors occupies a subset of the positive reals. This structure is motivated by a heavy-tailed analogue of the factorial $n$ !, called the factoid of $n$. Properties of the factoid suggested by computer explorations are shown to be valid. Two topics about the determination of the Zipf exponent in the rank-size law for city sizes are discussed.

Keywords: Stopped random product; heavy tail; limit theorem; renewal theory; convolution equivalence; Zipf law; Markov chain; invariant measure

2000 Mathematics Subject Classification: Primary 60E05; 60F05; 60G50; 60J05; 91 D10
\end{abstract}

\section{Introduction}

There is presently intense interest in stochastic models which give rise to heavy-tailed probability laws. Reviews with many references include Adler et al. (1998), Mitzenmacher (2004), Newman (2005), and Rachev (2003). Reed and Hughes (2002) showed how heavytailed laws can be obtained from random products $\prod_{j=1}^{N} X_{j}$, where the factors are independent copies of a random variable $X>0$, and $N$ is positive integer valued and independent of $\left\{X_{j}\right\}$. They showed (in subsequent papers) that certain choices of the generating laws yield explicit heavy-tailed laws which fit well to data arising from diverse areas. In this paper we examine some random products where $N$ is a hitting time derived from the sequence of factors.

The specific model which suggests this structure is the random version of the factorial $n$ ! due to Hayes (2007a). Fix an integer $n \geq 2$, and let $X_{1}, X_{2}, \ldots$ be independent with the uniform law on the set $\{1,2, \ldots, n\}$. Let $N=\inf \left\{j \geq 1: X_{j}=1\right\}$. Hayes defined the factoid of $n$ by $n ?=\prod_{j=1}^{N} X_{j}$. As $n$ increases, $n$ ? can be expected to grow somewhat like $n$ ! because (i) the mean of random factors is $\frac{1}{2}(n+1)$, the same as the average value of the factors of $n !$; and (ii) $\mathrm{E}[N]=n$. By using simulation experiments with $n=10$, Hayes (2007a) concluded that $n$ ? has a heavy right-hand tail, certainly heavier than a lognormal. In addition, $\mathrm{E}[n$ ?] appeared to be undefined, i.e. not finite. A histogram constructed from his experimental data suggested that $\lim _{n \rightarrow \infty} \mathrm{P}(n ?>n !)=\mathrm{e}^{-1}$, a conjecture which he verified analytically. Contributions to Hayes' weblog (Hayes (2007b)) raise the question of alterations to these findings if the stopping rule is changed to a different 'sentinel value', $i \neq 1$.

One purpose of this paper is an analytical investigation of the properties of $n$ ? and some related models. We begin in Section 2 by presenting our general formulation of more general stopped random products and showing how it reduces to a study of random sums stopped at an independent geometrically distributed random time. A random sum limit theorem is used to give a general limit theorem for our random products.

Received 28 February 2008; revision received 15 October 2008.

* Postal address: School of Mathematics and Statistics, University of Western Australia, 35 Stirling Highway, Crawley, WA 6009, Australia. Email address: pakes@maths.uwa.edu.au 
Sections 3 and 4 are devoted to (a generalisation of) the factoid. We show in Section 3 that there is a critical exponent $\alpha(n)>0$ such that $n$ ? has finite moments of order $t$ if and only if $t<\alpha(n)$, and we study how the exponent behaves as $n \rightarrow \infty$. In Section 4 we find, in a sense to be made precise, that $\mathrm{P}(n ?>x)$ is comparable to $x^{-\alpha(n)}$ for large $x$.

A continuous version of $n$ ? is examined in Section 5. Specifically, the $X_{i}$ are identically distributed with an arbitrary law which is supported in the nonnegative half-line, and the product is stopped at the first factor which hits the interval $(0, \zeta)$, where $\zeta$ is a fixed positive number. We develop several factoidal-type properties. The asymptotic régime $n \rightarrow \infty$ for the factoid is replaced by $\zeta \rightarrow 0$.

Gabaix (1999) presented several interesting multiplicative models to explain the Zipf law for city sizes, i.e. the power-law relation between absolute size and size rank. His mathematical details are obscure in places. In Section 6 we discuss two topics arising from his paper. The first concerns the determination of the Zipf exponent $a$ for a model which incorporates a minimum allowable size $s_{\min }$, and Gabaix's determination of $a$ as $\left(1-s_{\min } / \bar{s}\right)^{-1}$, where $\bar{s}$ is the mean size of cities. This identification is approximately valid under a 'heavy-traffic' regime of an underlying random walk, but it is not valid in general.

The second topic is the sense in which a Zipf exponent can be associated with the product Markov chain $\left(S_{n}: n=0,1, \ldots\right)$, where $S_{n}=\prod_{j=1}^{n} X_{j}$. This turns on identifying its invariant measures, and we find that there is an association with $a=1$ independently of the form of the law of $X$.

There is some duplication of notation between sections, but no confusion will result from this.

\section{General formulation}

Throughout the paper, let $X$ be a positive random variable with a nondegenerate law, and let $X_{1}, X_{2}, \ldots$ be independent copies of $X$. Denote the distribution function of $X$ by $F$, and its support by $\operatorname{supp}(F)$. Let $\mathcal{A}$ be a proper subset of $\operatorname{supp}(F)$ such that $p:=P(X \in \mathcal{A}) \in(0,1)$. The random variable $N=\inf \left\{j: X_{j} \in \mathcal{A}\right\}$ is a stopping time with respect to the sequence $\left\{X_{j}: j \geq 1\right\}$, and it has the geometric law

$$
\mathrm{P}(N=j)=(1-p)^{j-1} p, \quad j \geq 1, \quad \text { and } \quad \mathrm{E}[N]=p^{-1} .
$$

Define the product $\Pi=\prod_{j=1}^{N-1} X_{j}$, where $\Pi=1$ if $N=1$. If $B_{1}, B_{2}, \ldots$ are Borel subsets of $(0, \infty)$ then

$$
\mathrm{P}\left(X_{1} \in B_{1}, \ldots, X_{j-1} \in B_{j-1}, N=j\right)=F(\mathcal{A}) \prod_{i=1}^{j-1} F\left(B_{j} \backslash \mathcal{A}\right),
$$

where the product is taken as unity if $j=1$. It follows that

$$
\mathrm{P}\left(X_{1} \in B_{1}, \ldots, X_{j-1} \in B_{j-1} \mid N=j\right)=\prod_{i=1}^{j-1} \frac{F\left(B_{j} \backslash \mathcal{A}\right)}{1-p},
$$

i.e. the factors of $\Pi$ are conditionally independent given $N$.

The conditional distribution function of $X$, given $X \notin \mathcal{A}$, is

$$
G_{p}(x)=\frac{F((0, x] \backslash \mathcal{A})}{1-p} .
$$


Let $Y$ be a generic random variable having this distribution function, and let $Y_{1}, Y_{2}, \ldots$ be independent copies of $Y$, and independent of $N$. Then

$$
\Pi \stackrel{\mathrm{D}}{=} \prod_{j=1}^{N-1} Y_{j}
$$

where ' $\stackrel{\text { D }}{=}$ denotes equality in distribution. In particular, if $U_{j}=\log Y_{j}$ then

$$
\log \Pi \stackrel{\mathrm{D}}{=} \sum_{j=1}^{N-1} U_{j}
$$

where an empty sum is defined to be 0 . The right-hand side is a geometric sum of the kind studied in Gnedenko and Korolev (1996) and Kalashnikov (1997). Limit theorems for $\log \Pi$ can in principle be deduced as immediate consequences of the double-array transfer theorems described in Gnedenko and Korolev (1996, Chapter 4).

Specifically, suppose that $F$ depends on a positive-valued parameter $\zeta$ and that $p(\zeta):=$ $p \rightarrow 0$ as $\zeta \rightarrow 0$. If we denote $N$ by $N_{\zeta}$ and let $n(\zeta)=[1 / p(\zeta)]$, then it is evident that $N_{\zeta} / n(\zeta) \stackrel{\mathrm{D}}{\rightarrow} \varepsilon$, where $\varepsilon$ has the standard exponential law $\operatorname{Exp}(1)$ and $\stackrel{\text { 'D }}{\rightarrow}$ ' denotes convergence in distribution. Denote $U_{j}$ by $U_{\zeta, j}$ to emphasise the dependence of $G_{p}$ on $\zeta$. Thus, we have an indexed family of sequences $\left\{\left(U_{\zeta, j}: j=1,2, \ldots\right): \zeta>0\right\}$ and random products $\Pi_{\zeta}$. Let $k(\zeta)$ be a family of positive constants such that if $S_{\zeta, j}=\sum_{i=1}^{j-1} U_{\zeta, i} / k(\zeta)$ then there is a nonnegative random variable $V$ such that, as $\zeta \rightarrow 0$,

$$
S_{\zeta, n(\zeta)} \stackrel{\mathrm{D}}{\rightarrow} V .
$$

Theorem 4.1.1 of Gnedenko and Korolev (1996) holds with their sequences $\left\{a_{n}\right\}$ and $\left\{b_{n}\right\}$ identically 0 . Thus, we obtain the following result.

Theorem 2.1. If $p(\zeta) \rightarrow 0$ and (2.3) both hold as $\zeta \rightarrow 0$ then

$$
\frac{\log \Pi_{\zeta}}{k(\zeta)} \stackrel{\mathrm{D}}{\rightarrow} \varepsilon V
$$

and the factors in the limit are independent. The limit law is a mixture of exponential laws and, hence, it is infinitely divisible.

In another direction, denote the distribution function of $U_{1}$ by $J_{p}(y)=G_{p}\left(\mathrm{e}^{y}\right)$, and its $j$-fold convolution by $J_{p}(y ; j)$. Denote the survivor function of a distribution function, of $F$ say, by $\bar{F}=1-F$. It follows from (2.1) that

$$
\mathrm{P}\left(\log \Pi_{\zeta}>y\right)=p \sum_{j \geq 1}(1-p)^{j-1} \bar{J}_{p}(y ; j-1) .
$$

In principle, the asymptotic form of the left-hand side as $x \rightarrow \infty$ can be deduced from this, or from related identities. 


\section{The factoid}

As foreshadowed in Section 1, we fix integers $n \geq 2$ and $i \in \wp_{n}:=\{1,2, \ldots, n\}$, and let $X$ have the uniform law on $s_{n}$. Define

$$
n ? i=\prod_{j=1}^{N-1} X_{j},
$$

where $N=\inf \left\{j: X_{j}=i\right\}$. The case $n=2$ is trivial since $\mathrm{P}(2 ? 2=1)=1$ and $\mathrm{P}(2 ? 1=$ $\left.2^{j-1}\right)=2^{-j}$. The latter relation is essentially the probability law of the payoff in the Petersberg game.

Taking $\mathcal{A}=\{i\}$ in Section 2, we see that $Y$ has the uniform law on $\wp_{n} \backslash\{i\}$. In particular, if $t$ is real then $\mathrm{E}\left[Y^{t}\right]=M(t ; n, i) /(n-1)$, where

$$
M(t ; n, i)=\sum_{1 \leq j \leq n, j \neq i} j^{t} .
$$

In the notation of Section 2, the parameter $\zeta$ here is $n^{-1}$ and $p=\zeta$. It follows from (2.1) that

$$
\begin{aligned}
\mu(t ; n, i) & :=\mathrm{E}\left[(n ? i)^{t}\right] \\
& =n^{-1} \sum_{j \geq 1}\left(1-n^{-1}\right)^{j-1}\left(\mathrm{E}\left[Y^{t}\right]\right)^{j-1} \\
& =\frac{1}{n-M(t ; n, i)} .
\end{aligned}
$$

Our first result concerns the existence of moments.

Theorem 3.1. If $n \geq 2$ and $1 \leq i \leq n$, there exists positive constants $\alpha(n, i)$ such that $\mu(t ; n, i)<\infty$ if and only if $-\infty<t<\alpha(n, i)$. Moreover, if $n \geq 3$ then

$$
\alpha(n, 1)<\alpha(n, 2)<\cdots<\alpha(n, n)=\alpha(n-1,1)<\cdots<\alpha(2,1)=1<\alpha(2,2)=\infty .
$$

Proof. The function $M(t ; n, i)$ is defined and strictly increasing on the real line, and

$$
\lim _{t \rightarrow-\infty} M(t ; n, i)=0 .
$$

In addition, $M(0 ; n, i)=n-1$ and $M(1 ; n, i)=\frac{1}{2} n(n+1)>n$. Hence, if $(n, i) \neq(2,2)$, the equation

$$
M(t ; n, i)=n
$$

has a unique solution $\alpha(n, i)$ in $(0,1]$. So the denominator in (3.1) is positive if $t<\alpha(n, i)$, and the first assertion follows from these properties. The inequalities in (3.2) follow because $M(t ; n, i)$ decreases as $i$ increases, and the equality $\alpha(n, n)=\alpha(n-1,1)$ follows because the equation $M(t ; n, n)=n$ is the same as $M(t ; n-1,1)=n-1$.

Table 1 exhibits the critical exponents $\alpha(n, i)$ for $n \leq 5$. Theorem 3.1 immediately shows that $n$ ? $i$ has a heavy upper tail. It is clear from (3.3) that $t_{n}:=\alpha(n, 1) \rightarrow 0$ as $n \rightarrow \infty$, and, hence, the linear order specified by (3.2) implies that $\alpha(n, i) \rightarrow 0$ uniformly with respect to $i \in \wp_{n}$. Our next result determines the speed of this convergence. 
TABLE 1: Illustrative values of the critical exponent $\alpha(n, i)$.

\begin{tabular}{ccccc}
\hline & \multicolumn{4}{c}{$n$} \\
\cline { 2 - 5 } & 2 & 3 & 4 & 5 \\
\hline 1 & 1 & 0.448 & 0.269 & 0.185 \\
2 & $\infty$ & 0.631 & 0.326 & 0.210 \\
3 & - & 1.000 & 0.382 & 0.231 \\
4 & - & - & 0.448 & 0.250 \\
5 & - & - & - & 0.269 \\
\hline
\end{tabular}

Theorem 3.2. We have $\lim _{n \rightarrow \infty}(n \log n) \alpha(n, i)=1$ uniformly with respect to $i \in \wp_{n}$.

Proof. Since $(n-1) \log (n-1) / n \log n \rightarrow 1$, the uniform convergence again follows from (3.2) once we show that $(n \log n) t_{n} \rightarrow 1$. To see this, note that

$$
\int_{j-1}^{j} x^{t} \mathrm{~d} x<j^{t}<\int_{j}^{j+1} x^{t} \mathrm{~d} x
$$

and sum over $j$ to obtain the bounds

$$
L_{n}(t):=\frac{n^{1+t}-1}{1+t}<M(t ; n, 1)<U_{n}(t):=\frac{(n+1)^{1+t}-2^{1+t}}{1+t} .
$$

The integral definition of the bounds shows that they are strictly increasing in $t$. Hence, the solution $t_{n}^{\prime}$ of $L_{n}(t)=n$ satisfies $t_{n}<t_{n}^{\prime}$. This equation is

$$
1+t=n^{t}-n^{-1}=\mathrm{e}^{t \log n}-n^{-1} .
$$

It follows that $t_{n}^{\prime} \rightarrow 0$ as $n \rightarrow \infty$, for if not, there is a subsequence $\left\{t_{n(j)}\right\}$ bounded away from 0 , and then the right-hand side of the equation for $n=n(j)$ grows unboundedly relative to the left-hand side as $j \rightarrow \infty$. It follows that $t_{n}^{\prime} \log n \rightarrow 0$. Expanding the exponential term and cancelling the constant term thus yields $t=t(\log n)(1+o(t))-n^{-1}$, i.e. $1+1 / n t=$ $(\log n)(1+o(1))$. It follows that $1 / n t_{n}^{\prime} \sim \log n$, i.e. $t_{n}^{\prime} \sim 1 / n \log n$.

The unique solution $t_{n}^{\prime \prime}$ of $n=U_{n}(t)$ satisfies $t_{n}^{\prime \prime}<t_{n}<t_{n}^{\prime}$. Write this equation as

$$
1+t=\left(1+\frac{1}{n}\right) \mathrm{e}^{t \log (n+1)}-\left(\frac{2}{n}\right)(1+o(t))
$$

and expand the exponential factor to obtain

$$
1+t=\left(1+\frac{1}{n}\right)\left(1+t \log n+O\left(n^{-2}\right)+O\left(\frac{t}{n}\right)\right)-\left(\frac{2}{n}\right)(1+o(1)),
$$

i.e. $t=t \log n-n^{-1}+O\left(n^{-2}\right)$. Since $t=O(1 / n \log n)$, we conclude that $t_{n}^{\prime \prime} \sim 1 / n \log n$. It follows that $t_{n} \sim 1 / n \log n$. This completes the proof.

We might have expected this asymptotic form of the $\alpha(n, i)$ because the leading term in Stirling's formula shows that $\log n ! \sim n \log n$. Comparing values of $1 / n \log n$ with those of $\alpha(n, 1)$ in Table 1 exhibits a poor approximation with such small values of $n$. Further analysis 
of the bounds used in the above proof shows that the asymptotic behaviour is approached rather slowly; $\alpha(n, 1) n \log n-1 \sim 1 / \log n$.

One measure of 'typical value' of $n$ ? $i$ is its geometric mean $g(n, i)$. Applying Wald's lemma for random sums to (2.2) yields

$$
\mathrm{E}[\log (n ? i)]=\mathrm{E}[N-1] \mathrm{E}[\log Y]=\sum_{j=1, j \neq i}^{n} \log i=\log \left(\frac{n !}{i}\right) .
$$

It follows that $i g(n, i)=n$ !, independent of $i$. According to this measure, if we redefine the generalised factoid product to include the sentinel value $i$, this measure of typical value is independent of the stopping rule.

Another measure of 'typical value' is the median, $m(n, i)$. It seems possible to obtain only a rather rough estimate of $m(n, i)$ for large $n$. This comes from the following limit theorem.

Theorem 3.3. Let $\lambda(n, i)=\log (n$ ? $i)$. Then $\lambda(n, i) / n \log n \stackrel{\mathrm{D}}{\rightarrow} \varepsilon$ uniformly with respect to $i \in \delta_{n}$, where $\varepsilon \sim \operatorname{Exp}(1)$.

Proof. Begin by observing that, for $\ell=1,2, \ldots$,

$$
\mathrm{E}\left[U^{\ell}\right]=(n-1)^{-1}\left(\sum_{j=2}^{n}(\log j)^{\ell}-(\log i)^{\ell}\right) .
$$

It follows that $\lim _{n \rightarrow \infty} \mathrm{E}\left[(U / \log n)^{\ell}\right]=1$ uniformly with respect to $i \in \wp_{n}$. In the notation of Section 2 , let $n(\zeta)=n-1$ and $k(\zeta)=n \log n$, and write

$$
T_{n}=S_{\zeta, n(\zeta)}=\sum_{j=1}^{n-1} \frac{U_{j}}{k(n)}
$$

If $\delta>0$ then Markov's inequality asserts that $\mathrm{P}\left(\left|T_{n}-1\right|>\delta\right) \leq \delta^{-2} \mathrm{E}\left[\left(T_{n}-1\right)^{2}\right]$. The expectation equals

$$
\frac{(n-1) \mathrm{E}\left[U^{2}\right]+(n-1)(n-2)(\mathrm{E}[U])^{2}}{(n \log n)^{2}}-\frac{2(n-1) \mathrm{E}[U]}{n \log n}+1,
$$

and we now see that this quantity tends to 0 uniformly with respect to $i$. It follows that (2.1) holds with $V=1$, uniformly with respect to $i \in \&$, and the assertion now follows from Theorem 2.1.

Stirling's formula implies that $\log n !=(n \log n)(1+o(1))$ and, hence, Theorem 3.3 can be expressed as

$$
\lim _{n \rightarrow \infty} \mathrm{P}\left(\frac{\log (n ? i)}{\log (n !)}>x\right)=\mathrm{e}^{-x} .
$$

This generalises the result of Hayes' (2007a) simulation and calculation for the case in which $i=x=1$. Combining the identity

$$
\mathrm{P}\left(\frac{\log (n ? i)}{\log (n !)}>\frac{\log m(n, i)}{\log (n !)}\right)=\frac{1}{2}=\mathrm{e}^{-\log 2}
$$

with Theorem 3.3 yields a crude estimate of the median as $\log m(n, i) \sim 0.693 \log n$ !. This yields the estimate $m(10,1) \approx 35200$, rather more than Hayes' estimated value of 27000 . 


\section{Factoid tails}

Let $S(x ; n, i)=\mathrm{P}(n ? i>x)$ denote the survivor function of the factoid. The asymptotic behaviour of this function can be elucidated with the help of some renewal theory. Let $T(y ; n, i)$ be the survivor function of $\lambda(n, i)=\log (n ? i)$. There are two atypical examples where $\lambda(n, i)$ has a lattice law.

Example 4.1. We have seen that $\mathrm{P}\left(2 ? 1=2^{j}\right)=2^{-j-1}$ for $j=0,1, \ldots$ Hence,

$$
T(y ; 2,1)=2^{-j-1} \quad \text { if } \quad j \log 2 \leq y<(j+1) \log 2 .
$$

If we write $y=j \log 2+\delta(y)$ for such $y$ then $0 \leq \delta(y)<\log 2$ and $T(y ; 2,1)=\frac{1}{2} \exp (-y+$ $\delta(y))$. It follows that $S(x ; 2,1)=(2 x)^{-1} \exp (\delta(\log x))$. In particular,

$$
(2 x)^{-1} \leq S(x ; 2,1)<x^{-1},
$$

and, hence, 2 ? 1 can be said to have a weak power law with exponent equal to -1 .

Example 4.2. Clearly 4 ? 3 takes values which are powers of 2 . The equation which determines $\alpha(4,3)$ is $3-2^{t}-4^{t}=0$, a quadratic equation for $2^{t}$. Algebra yields $3-x-x^{2}=$ $\left(x_{1}-x\right)\left(x_{2}+x\right)$, where

$$
x_{1}=\frac{1}{2}(\sqrt{13}-1)=1.3028 \text { and } x_{2}=\frac{1}{2}(\sqrt{13}+1)=2.3028 .
$$

The Laplace-Stieltjes transform of $\lambda(4,3)$ is $\mu(-s)$, whence

$$
\mu(-s)=\frac{1}{\sqrt{13}}\left(\frac{1}{x_{1}-x}+\frac{1}{x_{2}+x}\right),
$$

where $x=2^{-s}$. Expanding as a power series in $x$ yields

$$
\mathrm{P}(\lambda(4,3)=j \log 2)=\frac{x_{1}^{-j-1}+\left(-x_{2}\right)^{-j-1}}{\sqrt{13}},
$$

and, hence,

$$
T(j \log 2 ; 4,3)=\frac{1}{\sqrt{13}}\left(\frac{x_{1}^{-j}}{x_{1}-1}+\frac{\left(-x_{2}\right)^{-j}}{x_{2}+1}\right) .
$$

So, finally, if $\delta(y)$ is as defined above then

$$
\begin{aligned}
T(y ; 4,3) & =\frac{1}{\sqrt{13}}\left(\frac{x_{1}^{-(y-\delta(y)) / \log 2}}{x_{1}-1}+\frac{\left(-x_{2}\right)^{-(y-\delta(y)) / \log 2}}{x_{2}+1}\right) \\
& =\frac{1}{\sqrt{13}}\left[\frac{1}{x_{1}-1} \exp \left(-\frac{\log x_{1}}{\log 2}(y-\delta(y))\right)+O\left(\exp \left(-y \frac{\log x_{2}}{\log 2}\right)\right)\right] \\
& =0.9160 \mathrm{e}^{-0.3816(y-\delta(y))}+O\left(\mathrm{e}^{-1.2034 y}\right) .
\end{aligned}
$$

Since $\alpha(4,3)=\log x_{1} / \log 2=0.3816$, it follows that

$$
S(x ; 4,3)=0.9160 x^{-\alpha(4,3)} \mathrm{e}^{\alpha(4,3) \delta(\log x)}+O\left(x^{-1.1034}\right),
$$

and, hence,

$$
0.9160=\liminf _{x \rightarrow \infty} x^{\alpha(4,3)} S(x ; 4,3)<1.1933=\limsup _{x \rightarrow \infty} x^{\alpha(4,3)} S(x ; 4,3) .
$$

If $(n, i) \neq(2,1),(4,3)$ then it is evident that $\lambda(n, i)$ has a nonlattice discrete law. Consequently, we might expect that a more precise result exists. Let $A(n, i)=M^{\prime}(\alpha(n, i) ; n, i)$. 
Theorem 4.1. If $(n, i) \neq(2,1),(4,3)$ then

$$
\lim _{x \rightarrow \infty} x^{\alpha(n, i)} S(x ; n, i)=\frac{1}{A(n, i) \alpha(n, i)} .
$$

Proof. We will simplify the notation by omitting the argument $(n, i)$, in which case (3.1) becomes $\mu(t)=(n-M(t))^{-1}$. The notation is further simplified by writing $\kappa$ for $\alpha(n, i)$, so that $A(n, i)=M^{\prime}(\kappa)$. The analytical structure of renewal theory can be invoked by writing

$$
n \mu(-s)=n \mathrm{E}\left[\mathrm{e}^{-s \lambda}\right]=\frac{n}{n-M(-s)}=\frac{1}{1-\ell(s)},
$$

where $\ell(s)=\int_{0}^{\infty} \mathrm{e}^{-s y} L(\mathrm{~d} y)$ and $L(\mathrm{~d} y)$ is the defective probability law which assigns mass $n^{-1}$ to $\log j$ for $j=1, \ldots, n$ but $j \neq i$. It follows that

$$
\mathrm{P}(\lambda \leq y)=\left(1-L_{\infty}\right) \sum_{k \geq 0} L^{* k}(y),
$$

where $L^{* k}$ denotes the $k$-fold convolution of the distribution function $L$ and $L_{\infty}=L(\infty)=$ $1-n^{-1}$.

Following the notation in Feller (1971, p. 366), define the exponentially tilted distribution function $L^{\#}(\mathrm{~d} y)=\mathrm{e}^{\kappa y} L(\mathrm{~d} y)$, whose first moment is

$$
\mu^{\#}=\int_{0}^{\infty} y \mathrm{e}^{\tau y} L(\mathrm{~d} y)=n^{-1} \sum_{j \neq i} j^{\tau} \log j=\frac{A(n, i)}{n} .
$$

It follows from Feller (1971, p. 377) that

$$
\lim _{y \rightarrow \infty} \mathrm{e}^{\kappa y} \mathrm{P}(\lambda>y)=\frac{1-L_{\infty}}{\kappa \mu^{\#}}=(\kappa A(n, i))^{-1},
$$

and the assertion follows.

Hayes (2007a) obtained the numerical estimate 0.07 for $\alpha(10,1)$. This is too high, as $M(0.07 ; 10,1)=10.13$. Numerical calculation shows that $0.0624<\alpha(10,1)<0.0625$ and $M(0.0625 ; 10,1)=10.00027$. The asymptotic result of Theorem 3.2 can be recast as $\lim _{n \rightarrow \infty} \alpha(n, i) \log n !=1$. Numerical results give some credence to the conjecture that

$$
(1+\log n !)^{-1}<\alpha(n, 1)<(\log n !)^{-1} .
$$

For example, this inequality yields $0.0621<\alpha(10,1)<0.0662$.

We know that $\mathrm{E}\left[(n ? i)^{\alpha(n, i)}\right]=\infty$. Details of the proof of Theorem 4.1 allow us to estimate the 'speed of divergence' of this moment. Let

$$
A_{2}(n, i)=M^{\prime \prime}(\alpha(n, i))=\sum_{j \neq i}(\log j)^{2} j^{\alpha(n, i)} .
$$

Theorem 4.2. If $(n, i) \neq(2,1),(4,3)$ then

$$
\lim _{x \rightarrow \infty}\left[\mathrm{E}\left[(n ? i)^{\alpha(n, i)} ; n ? i \leq x\right]-(A(n, i))^{-1} \log x\right]=\frac{A_{2}(n, i)}{2 A^{2}(n, i)} .
$$


Proof. Using the notation in the proof of Theorem 4.1, we see from its definition that the Laplace-Stieltjes transform of $L^{\#}$ is, for $s>0$, equal to $\ell(s-\kappa)$. It follows from (4.1) that the Laplace-Stieltjes transform of the renewal function $U$ generated by $L^{\#}$ is $n \mu(\kappa-s)$, i.e.

$$
n \mathrm{E}\left[\mathrm{e}^{\kappa \lambda} ; \lambda \leq y\right]=U(y)
$$

Since $L^{\#}$ has a finite second-order moment, it follows from the refined elementary renewal theorem (Feller (1971, p. 366)) that $U(y)-y / \mu^{\#} \rightarrow \int_{0}^{\infty} y^{2} L^{\#}(\mathrm{~d} y) / 2\left(\mu^{\#}\right)^{2}$. The second-order moment equals $A_{2}(n, i) / n$ and the assertion follows.

\section{A small factor stopping rule}

In the notation of Section 2 , let $\mathcal{A}=[0, \zeta]$, let $F(0)=0$, and let $F(x)>0$ if $x>0$. The random factors $X_{i}$ can be interpreted as independent price relatives, in which case $\Pi_{\zeta}$ is the value of an initial unit investment immediately before the time $N_{\zeta}$ of the first 'crash'. Thus, $p=F(\zeta)$, and writing $G_{\zeta}$ for $G_{p}$ we have

$$
G_{\zeta}(x)=\frac{F(x)-F(\zeta)}{1-F(\zeta)}
$$

and $G_{\zeta}(x) \rightarrow F(x)$ as $\zeta \rightarrow 0$.

If $\lambda:=\mathrm{E}[\log X]$ is finite then, in the notation of Theorem 2.1, choosing $k(\zeta)=n(\zeta)$ we see that $S_{\zeta, n(\zeta)} \stackrel{\mathrm{D}}{\rightarrow} \lambda$. It follows that

$$
F(\zeta) \log \Pi_{\zeta} \stackrel{\mathrm{D}}{\rightarrow} \lambda \varepsilon, \quad \zeta \rightarrow 0
$$

In the case in which $\lambda=0$, letting $k(\zeta)=\sqrt{n(\zeta)}$, it follows from the Lindeberg condition for triangular arrays that (2.1) holds with $V=\sigma_{l} Z$, where $\sigma_{l}^{2}=\operatorname{var}(\log X)$, assumed finite, and $Z$ has the standard normal law. Theorem 2.1 then gives the following result.

Lemma 5.1. If $\lambda=0$ and $\sigma_{l}^{2}<\infty$ then

$$
\sqrt{F(\zeta)} \log \Pi_{\zeta} \stackrel{\mathrm{D}}{\rightarrow} \sigma_{l} \varepsilon Z
$$

and the limit has the Cauchy law with characteristic function $\left(1+\frac{1}{2} \sigma_{l}^{2} t^{2}\right)^{-1}$.

In terms of the financial interpretation, it is of interest to consider the stopped product $\mathcal{P}_{\zeta}=\prod_{j=1}^{N_{\zeta}} X_{j}$. The derivation of (2.1) shows that $\mathcal{P}_{\zeta} \stackrel{\mathrm{D}}{=} \prod_{\zeta} V_{\zeta}$, where $V_{\zeta}$ is independent of $\Pi_{\zeta}$ with distribution function $\mathrm{P}(X \leq x \mid X \leq \zeta)=F(x \wedge \zeta) / F(\zeta)$. If $0<y \leq 1$ then

$$
\mathrm{P}\left(\frac{V_{\zeta}}{\zeta} \leq y\right)=\frac{F(\zeta y)}{F(\zeta)}
$$

and the right-hand side has a limit as $\zeta \downarrow 0$ if and only if there is a constant $\delta \geq 0$ and a slowly varying function $\ell$ such that $F(x)=x^{\delta} \ell(1 / x)$. In this case, $V_{\zeta} / \zeta \stackrel{\mathrm{D}}{\rightarrow} W$, where $\mathrm{P}(W \leq y)=(y \wedge 1)^{\delta}$. So if $\delta>0$ then, in the limit $\zeta \downarrow 0, \mathcal{P}_{\zeta}$ equals $\Pi_{\zeta}$ scaled down by an independent factor $\zeta W$. It follows that $\log \mathcal{P}_{\zeta}$ has the same limit law as $\log \Pi_{\zeta}$. 
Next we examine the moment function of $\Pi_{\zeta}$, which is computed as

$$
\begin{aligned}
M(t ; \zeta) & =\sum_{n \geq 1} \mathrm{E}\left[\Pi_{\zeta}^{t} ; N_{\zeta}=n\right] \\
& =\sum_{n \geq 1} p(1-p)^{n-1}\left(\int_{0}^{\infty} x^{t} \mathrm{~d} G_{\zeta}(x)\right)^{n-1} \\
& =p \sum_{n \geq 1}\left(m_{\zeta}(t)\right)^{n-1}
\end{aligned}
$$

where $m_{\zeta}(t)=\int_{\zeta}^{\infty} x^{t} \mathrm{~d} F(x)$ and the integral is understood to be over $(\zeta, \infty)$. Write $m(t)=$ $m_{0}(t)$, and let $\tau=\sup \{t \geq 0: m(t)<\infty\}$. The following is obvious.

Lemma 5.2. If $t>0$ then the moment function $M(t ; \zeta)<\infty$ if and only if $\tau>0$ and $m_{\zeta}(t)<1$. In this case,

$$
M(t ; \zeta)=\frac{p}{1-m_{\zeta}(t)} .
$$

The conditions of the lemma are satisfied for all $t$ if $F(1)=1$ because $m_{\zeta}(t) \leq m(t)<1$. In this case,

$$
\lim _{t \rightarrow \infty} M(t ; \zeta)=\frac{p}{\mathrm{P}\left(X_{1} \neq 1\right)}
$$

In the sequel we assume that $\tau>0$ and that $F(1)<1$, i.e. that $r_{F}:=\sup \{x: F(x)<1\}>1$. Note that $m_{\zeta}(t)$ is continuous in $(0, \tau)$ and that it is possible to have $m(\tau)<\infty$. To see this, choose any $\tau>0$ and $L(x)$ slowly varying at $\infty$ such that $\int_{1}^{\infty} x^{-1} L(x) \mathrm{d} x<\infty$. There exist density functions $f(x)$ proportional to $x^{-1-\tau} L(x)$ if $x \geq 1$.

To proceed further, observe that

$$
m^{\prime \prime}(t)=\int_{0}^{\infty}(\log x)^{2} x^{t} \mathrm{~d} F(x)
$$

is finite if $0<t<\tau$. Hence, each $m_{\zeta}$ is convex in $(0, \tau)$ and if $\zeta \in\left(0, r_{F}\right)$ then $m_{\zeta}(0)=$ $1-p<1$. Finally, $m_{\zeta}(t) \uparrow m(t)$ as $\zeta \downarrow 0$. The next result follows because $m_{\zeta}^{\prime}(t)=$ $\int_{\zeta}^{\infty}(\log x) x^{t} \mathrm{~d} F(x)$ is finite for all $\zeta>0$ and $t \in(0, \tau), m_{\zeta}^{\prime}(0)$ is continuous at $\zeta=1$, and it is positive if $1<\zeta<r_{F}$. Recall that $\lambda=\mathrm{E}[\log X]$, and note that $\lambda<\infty$ since $\tau>0$.

Lemma 5.3. Suppose that $0<t<\tau$ and $0 \leq \zeta<r_{F}$. If $\lambda \geq 0$ then $m_{\zeta}$ is convex increasing in $(0, \tau)$. If $\lambda<0$ then there exists a number $\zeta^{\prime} \in(0,1)$ such that $m_{\zeta}^{\prime}(0)>0$ or $m_{\zeta}^{\prime}(0)<0$ if $\zeta^{\prime}<\zeta<r_{F}$ or, respectively, $0<\zeta<\zeta^{\prime}$.

So if $m(\tau) \leq 1$ then $M(t ; \zeta)$ is finite or infinite for all $0<\zeta<r_{F}$ and $t \leq \tau$ or, respectively, $t>\tau$. This suggests the possibility that $\Pi_{\zeta}$ and $X$ are tail equivalent, and we examine this in Theorem 5.2, below.

The more typical case is where

$$
m(\tau)>1,
$$

where we understand that $m(\tau)=\infty$ if $\tau=\infty$. If $m(\tau)=\infty$ then $m_{\zeta}(\tau)=\infty$ for all $\zeta \in\left(0, r_{F}\right)$. Suppose that $1<m(\tau)<\infty$. Since $F$ is not degenerate, it is clear that $m_{\zeta}(\tau)>1$ or $m_{\zeta}(\tau)<1$ if $\zeta$ is sufficiently close to 0 or, respectively, $r_{F}$. In more detail, since $m_{\zeta}(\tau)$ is a left-continuous function of $\zeta$, it follows that there exists a number $\xi \in\left(0, r_{F}\right)$ such 
that $m_{\zeta}(\tau)>1$ or $m_{\zeta}(\tau) \leq 1$ if $0<\zeta<\xi$ or, respectively, $\xi \leq \zeta<r_{F}$. So if $\zeta \geq \xi$ then $m_{\zeta}(t)<1$ for all $t<\tau$, which is a case covered by Lemma 5.2.

Define $\xi=0$ if $m(\tau)=\infty$. In the sequel we assume that $\zeta>\xi$, i.e. that

$$
m_{\zeta}(\tau)>1
$$

The following result is an obvious consequence of Lemma 5.3 and the continuity of $m_{\zeta}$.

Lemma 5.4. If $\tau>0$ and (5.3) holds then there exists a critical exponent $0<\alpha(\zeta) \leq \tau$ such that

$$
m_{\zeta}(\alpha(\zeta))=1
$$

and $M(t ; \zeta)<\infty$ if and only if $t<\alpha(\zeta)$. In addition, $\alpha(\zeta)$ is nondecreasing in $\zeta$.

Part (i) of Theorem 5.1, below, which concerns how $\alpha(\zeta)$ behaves as $\zeta \downarrow 0$, is a consequence of graphical considerations based on the convexity properties listed in Lemma 5.3 and of observing that (5.2) implies (5.3) if $\zeta$ is small enough.

Theorem 5.1. Suppose that $0<\tau \leq \infty$ and that (5.2) holds.

(i) If $\lambda \geq 0$ then $\alpha(\zeta) \downarrow \alpha:=0$ as $\zeta \downarrow 0$. If $\lambda<0$ then $\alpha(\zeta) \downarrow \alpha=\alpha(0)$, where $\alpha$ is the unique positive solution of $m(t)=1$.

(ii) If $\lambda \neq 0$ then

$$
\alpha(\zeta)-\alpha \sim \frac{\phi(\zeta)}{m^{\prime}(\alpha)}, \quad \zeta \downarrow 0,
$$

where $\phi(\zeta)=\int_{0}^{\zeta} x^{\alpha} \mathrm{d} F(x)$.

(iii) If $\lambda=0$ and $m^{\prime \prime}(0)<\infty$ then

$$
\alpha(\zeta) \sim \sqrt{\frac{2 \phi(\zeta)}{m^{\prime \prime}(0)}} .
$$

Proof. Observe that $m^{\prime}(\alpha)=\int_{0}^{\infty}(\log x) x^{\alpha} \mathrm{d} F(x)$ is finite and positive if $\lambda \neq 0$, since $m(t)$ is strictly increasing at $t=\alpha$. The identity $m_{\zeta}(\alpha(\zeta))=m(\alpha)=1$ can be expressed as

$$
\phi(\zeta)=\int_{\zeta}^{\infty}\left(x^{\alpha(\zeta)}-x^{\alpha}\right) \mathrm{d} F(x) .
$$

The integrand equals

$$
x^{\alpha}\left(\mathrm{e}^{(\alpha(\zeta)-\alpha) \log x}-1\right)=(\alpha(\zeta)-\alpha) x^{\alpha}\left(\log x+o(1)(\log x)^{2}\right),
$$

where the remainder term arises because $\alpha<\tau$. Integration yields $\phi(\zeta)=(\alpha(\zeta)-\alpha)\left(m^{\prime}(\alpha)+\right.$ $o(1)$ ), and (ii) follows since $\phi(\zeta) \rightarrow 0$. If $\lambda=0$ then $\alpha=0$ and the assertion follows in a similar way using the second-order expansion

$$
x^{\alpha(\zeta)}-1=\alpha(\zeta) \log x+\frac{1}{2} \alpha^{2}(\zeta)(\log x)^{2}(1+o(1) \log x) .
$$

We now fix $\zeta \in\left(0, r_{F}\right)$ and examine the survivor function $\bar{H}(x):=\mathrm{P}\left(\Pi_{\zeta}>x\right)$. Adding some technical conditions allows us to verify the tail equivalence of $\Pi_{\zeta}$ and $X$ in the case in which $m_{\zeta}(\tau)<1$. Let $J(y)=F\left(\mathrm{e}^{y}\right)$ and $J_{\zeta}(y)=\mathrm{P}(\log Y \leq y)=G_{\zeta}\left(\mathrm{e}^{y}\right)$. Denote the 
$n$-fold convolution power by, for example, $J^{* n}$, and integration over the real line is denoted simply as ' $\int$ '. Since

$$
\left(\int_{\zeta}^{\infty} x^{t} G_{\zeta}(\mathrm{d} x)\right)^{n}=\int \mathrm{e}^{t y} J_{\zeta}^{* n}(\mathrm{~d} y),
$$

it follows from (5.1) that

$$
\bar{H}\left(\mathrm{e}^{y}\right)=p(\zeta) \sum_{n \geq 1}(1-p(\zeta))^{n} \overline{J_{\zeta}^{* n}}(y) .
$$

Observing that $\tau$ is the abscissa of convergence of the moment generating function $\int \mathrm{e}^{t y} \mathrm{~d} J(y)$, we recall the definition of the convolution equivalence class $\delta_{\tau}$. Assume that $0 \leq \tau<\infty$ and that $r_{F}=\infty$. The distribution function $J$ has an exponential tail with rate $\tau$, written $J \in \mathcal{L}_{\tau}$, if, for all real $y$,

$$
\lim _{z \rightarrow \infty} \frac{\bar{J}(z-y)}{\bar{J}(z)}=\mathrm{e}^{\tau y} .
$$

If $J \in \mathcal{L}_{\tau}$ and $m(\tau)<\infty$, we say that $J$ is convolution equivalent, written $J \in \AA_{\tau}$, if

$$
\lim _{y \rightarrow \infty} \frac{\overline{J^{* 2}}(y)}{\bar{J}(y)}=2 m,
$$

where $m$ is finite.

See Pakes (2004) and the references therein for these concepts. A fundamental result of the theory is that $m=m(\tau)$. This result has long been known in the case of laws supported on the positive real line, although proofs in the early literature contain gaps. These are discussed in Foss and Korshunov (2007); see the remarks following their Theorem 4. Note, however, that the gap identified in Cline (1987, Lemma 2.3(ii)) (used to prove his Theorem 2.9) is claimed to be filled in Cline (1990), but this proof is faulty too. If $J$ is supported on the nonnegative half-line then, clearly, $\bar{J}(y) \leq \overline{J^{* n}}(y)$. The reverse of this inequality is implicitly used in line 6 of Cline's second proof. Albin (2008, Proposition 1.1) showed that Lemma 2.3(ii) of Cline (1987) is not true in the generality it claims, and he stated and proved a correct version of it (see Albin (2008, Theorem 3.1)). Foss and Korshunov (2007, Theorem 3) gave a gap-free proof that $m=m(\tau)$ for the positive support case using 'real analytic and probabilistic methods', and under lesser assumptions. Pakes (2004, Corollary 2.1) derived the general result from the positive support case.

Let $\mathscr{H}(y)$ denote the distribution function of $\log \Pi_{\zeta}$, so $\mathscr{H}(y)=H\left(\mathrm{e}^{y}\right)$.

Theorem 5.2. Suppose that $0<\zeta<r_{F}$ is fixed, that $J \in \mathscr{L}_{\tau}$, and that $m(\tau)<\infty$. If $m_{\zeta}(\tau)<1$ then $J \in \varsigma_{\tau}$ if and only if

$$
\lim _{x \rightarrow \infty} \frac{\bar{H}(x)}{\bar{F}(x)}=\frac{p(\zeta)}{(1-p(\zeta))\left(1-m_{\zeta}(\tau)\right)^{2}} .
$$

Each of these implies that $\mathcal{H} \in \wp_{\tau}$.

Proof. Observe that if $y>\log \zeta$ then $\bar{J}_{\zeta}(y)=\bar{J}(y) /(1-p(\zeta))$ and, hence, that $J_{\zeta} \in \mathcal{L}_{\tau}$. Moreover, if $J \in \delta_{\tau}$, it follows from Lemma 2.4(i) of Pakes (2004) that $J_{\zeta} \in \delta_{\tau}$. Identity (5.4) can be written as $\overline{\mathscr{H}}=\phi_{\zeta}\left(\bar{J}_{\zeta}\right)$, where $\phi_{\zeta}(s)=p(\zeta)(1-(1-p(\zeta)) a)^{-1}$ is the probability generating function of a geometric law. Our assumption that $m_{\zeta}(\tau)<1$ coincides with the 
condition expressed as $q M_{G}<1$ in Theorem 4.1 of Pakes (2007). It follows from this theorem that $\lim _{y \rightarrow \infty} \overline{\mathscr{H}}(y) / \bar{J}_{\zeta}(y)=\phi_{\zeta}^{\prime}\left(m_{\zeta}(\alpha(\zeta))\right)$ (correcting a typo in Pakes' statement). This is equivalent to (5.5). The other assertions follow directly from Theorem 4.1 of Pakes (2007).

Theorem 4.1 of Pakes (2007) asserts the converse that if $\mathscr{H} \in \delta_{\tau}$ then $J \in \delta_{\tau}$ and (5.5) holds. See also Watanabe (2008) for a proof of this. Finally, Lemma 2.3 of Pakes (2004) implies that $J \in \wp_{\tau}$ if

$$
\bar{F}(x)=x^{-\tau}\left(\exp \left(-c(\log x)^{\omega}\right)\right)(\log x)^{-\delta} L(\log x),
$$

where $c \geq 0, \omega<1, L$ is a normalised slowly varying function, and if $c=0$ then either $\delta>1$ or $\delta=1$ and $\int_{1}^{\infty}(L(y) / y) \mathrm{d} y<\infty$. Thus, the tail equivalence in Theorem 5.2 holds if $\bar{F}$ is regularly varying with index $\tau$ and with a restricted form of the slowly varying factor.

The proof of the following result for $m_{\zeta}(\tau) \geq 1$ rests on the standard gambit of exponential tilting followed by a renewal theorem.

Theorem 5.3. Suppose that $\zeta \in\left(0, r_{F}\right)$ is fixed and that either $m_{\zeta}(\tau)>1$ or $m_{\zeta}(\tau)=1$ and the one-sided derivative $m_{\zeta}^{\prime}(\alpha(\zeta))=m_{\zeta}^{\prime}(\tau-)$ is finite. If $J$ is nonlattice then

$$
\bar{H}(x) \sim C x^{-\alpha(\zeta)}, \quad x \rightarrow \infty,
$$

where $C=\left(\alpha(\zeta) m_{\zeta}^{\prime}(\alpha(\zeta))\right)^{-1}$.

Proof. Recall that $0<\alpha(\zeta)<\tau \leq \infty$ if $m_{\zeta}(\tau)>1$ and that $\alpha(\zeta)=\tau<\infty$ if $m_{\zeta}(\tau)=1$. Let $q=1-p(\zeta)$. The definition of $\alpha(\zeta)$ can be written as $q \int \mathrm{e}^{\alpha(\zeta) y} \mathrm{~d} J_{\zeta}(y)=1$, and, hence,

$$
K(y):=q \int_{-\infty}^{y} \mathrm{e}^{\alpha(\zeta) z} \mathrm{~d} J_{\zeta}(z)
$$

is a distribution function. We write the right-hand side of (5.4) as $p(\zeta) \sum_{n \geq 0} q^{n}\left(\bar{J} * J^{* n}\right)(y)$, and observing that $K^{* n}(y)=q^{n} \int_{-\infty}^{y} \mathrm{e}^{\alpha(\zeta) z} \mathrm{~d} J_{\zeta}^{* n}(z)$, a little algebra leads to the identity

$$
\mathrm{e}^{\alpha(\zeta) y} \bar{H}\left(\mathrm{e}^{y}\right)=p(\zeta) q \sum_{n \geq 0} \int \mathrm{e}^{\alpha(\zeta)(y-z)} \bar{J}_{\zeta}(y-z) \mathrm{d} K^{* n}(z)=p(\zeta) q \int \phi(y-z) \mathrm{d} U(z),
$$

where $\phi(y)=\mathrm{e}^{\alpha(\zeta) y} \bar{J}_{\zeta}(y)$ and $U(y)=\sum_{n \geq 0} K^{* n}(y)$ is a renewal function.

Note that $\phi(y)=0$ if $y<\zeta$ and that

$$
\phi(y)=\mathrm{e}^{\alpha(\zeta) y} \int_{y}^{\infty} \mathrm{d} J_{\zeta}(z) \leq \int_{y}^{\infty} \mathrm{e}^{\alpha(\zeta) z} \mathrm{~d} J_{\zeta}(z)
$$

It follows that the upper bound decreases to 0 as $y \uparrow \infty$ and that $\phi$ is bounded above by $q^{-1}$. Hence, $\phi$ is directly Riemann integrable, and if $z^{\prime}$ is real then the dominated convergence theorem implies that

$$
\lim _{y \rightarrow \infty} \int_{-\infty}^{z^{\prime}} \phi(y-z) \mathrm{d} U(z)=0
$$


The first-order moment

$$
\begin{aligned}
\mu & :=\int y \mathrm{~d} K(y) \\
& =\int \mathrm{e}^{\alpha(\zeta) y} \mathrm{~d} J_{\zeta}(y) \\
& =\frac{1}{q} \int_{\zeta}^{\infty}(\log x) x^{\alpha(\zeta)} \mathrm{d} F(x) \\
& =\frac{m^{\prime}(\alpha(\zeta))}{q}
\end{aligned}
$$

is positive, and it is finite since $\alpha(\zeta)<\infty$. A minor alteration of the details of the key renewal theorem (cf. Asmussen (2003, p. 300)) shows that

$$
\begin{aligned}
\int_{z^{\prime}}^{\infty} \phi(y-z) \mathrm{d} U(z) & =\frac{1+o(1)}{\mu} \int_{z^{\prime}}^{\infty} \phi(y-z) \mathrm{d} z \\
& =\frac{1+o(1)}{\mu} \int_{-\infty}^{y-z^{\prime}} \phi(u) \mathrm{d} u \\
& \rightarrow \mu^{-1} \int \phi(u) \mathrm{d} u, \quad y \rightarrow \infty
\end{aligned}
$$

The integral equals

$$
\begin{aligned}
\int \mathrm{e}^{\alpha(\zeta) y} \int_{y}^{\infty} \mathrm{d} J_{\zeta}(u) \mathrm{d} y & =(\alpha(\zeta))^{-1} \int \mathrm{e}^{\alpha(\zeta) u} \mathrm{~d} J_{\zeta}(u) \\
& =(\alpha(\zeta))^{-1} \int_{\zeta}^{\infty} x^{\alpha(\zeta)} \mathrm{d} G_{\zeta}(x) \\
& =\frac{m_{\zeta}(\alpha(\zeta))}{q \alpha(\zeta)} \\
& =(q m(\zeta))^{-1}
\end{aligned}
$$

The assertion follows.

\section{City sizes and Zipf's law}

Suppose that the population sizes of many cities are ranked in descending order of size, so that rank $r=1$ denotes the largest size, and so on. If $C(r)$ denotes the population size corresponding to rank $r$ then a consistent empirical finding is that $C(r) \propto r^{-a}$, where $a>0$. This relation is often called Zipf's law. In addition, the (Zipf) exponent $a$ is often close to unity. For example, Gabaix (1999) found that $a=1.005$ for the 135 largest US metropolitan areas in 1991. As an explanation, he postulated that the size of a city evolves in a multiplicative manner; thus, the size $S_{n}$ at time $n$ satisfies $S_{n+1}=S_{n} X_{n+1}$, where the $X_{n}$ are as in Section 2. This is Gibrat's law of proportionate effect. Gabaix recognised that this allows the possibility of arbitrarily small sizes. He avoided this by positing a lower bound $s_{\min }>0$ and modifying the above recursion to $S_{n+1}=S_{n} X_{n+1} \vee s_{\min }$; see Gabaix (1999, p. 749). Suppose that $F(0)=0$. If $W_{n}:=\log \left(S_{n} / s_{\min }\right)$ and $Z_{n}:=\log X_{n}$, then it is easy to check that $W_{n+1}=\left(W_{n}+Z_{n+1}\right) \vee 0$, a random walk reflected at the origin.

Noting that $\lambda=\mathrm{E}\left[Z_{n}\right]$, it is known that if $\lambda<0$ then this random walk has a limitingstationary law $\omega(y)=\mathrm{P}\left(W_{\infty} \leq y\right)$, where $W_{\infty}$ denotes the limit in law of $\left\{W_{n}\right\}$. Thus, the 
limit in law of the size process is $S_{\infty}=s_{\min } \exp \left(W_{\infty}\right)$, and its survivor function is

$$
\bar{\sigma}(x):=\mathrm{P}\left(S_{\infty}>x\right)=\mathrm{P}\left(W_{\infty}>\log x-\log s_{\min }\right) .
$$

The asymptotic behaviour of the right-hand side can be deduced from existing results about the random walk as follows. Let $R_{0}=0$ and $R_{n}=\sum_{j=1}^{n} Z_{j}(n \geq 1)$ denote the underlying unrestricted random walk, and let $M:=\sup _{n \geq 0} R_{n}$. A key result is that $W_{\infty} \stackrel{\mathrm{D}}{=} M$, and the tail behaviour of the supremum $M$ has been much studied in the queueing and random walk literature. The results we need are given the most coherent form in Korshunov (1997). In the notation of Section 5, $m(t)=\mathrm{E}\left[\exp \left(t Z_{1}\right)\right]$, so what he calls the Cramér case is the existence of the number $\alpha>0$ such that $m(\alpha)=1$ and the additional assumption that $m^{\prime}(\alpha)<\infty$. In this case, $\bar{\omega}(y) \sim C \mathrm{e}^{-\alpha y}$ as $y \rightarrow \infty$, where $C$ is a positive constant whose determination involves the law of the first ascending ladder height of the random walk $\left\{R_{n}\right\}$.

It follows that

$$
\bar{\sigma}(x) \sim C\left(\frac{x}{s_{\min }}\right)^{-\alpha}, \quad x \rightarrow \infty .
$$

In particular, the barrier $s_{\min }$ alters the constant of proportionality, but it has no effect on the tail Zipf exponent $a=\alpha$. This point is important because it contradicts Gabaix's determination of the exponent as $\left(1-s_{\min } / \bar{s}\right)^{-1}$, where $\bar{s}=\mathrm{E}\left[S_{\infty}\right]$. This expectation depends on the increment law in a very complicated way. However, the two determinations of the Zipf exponent can be reconciled within fairly narrow constraints, as we now explain.

Gabaix (1999) did not follow the above route; instead he replaced the random walk with the regulated Brownian motion (RBM) process,

$$
R_{t}:=\sup _{0 \leq s \leq t}\left(B_{t}-B_{s}\right),
$$

where $B_{t}=\sigma w_{t}-\mu, \mu, \sigma>0$, and $\left(w_{t}: t \geq 0\right)$ is a standard Brownian motion process. (Note that Gabaix wrote $\mu$ for our $-\mu$.) See Harrison $(1985$, p. 14) for the definition of the RBM process and the fact that it has a limiting/stationary law which is the exponential with mean $a^{-1}:=\sigma^{2} / 2 \mu$. The RBM process arises as the weak limit of a sequence of reflecting random walks with negative drifts which approach 0 . Gabaix used this exponential limit law in place of $\omega$, and this amounts to invoking a heavy-traffic approximation familiar in queueing theory. In its simplest terms, $m(t)$ is replaced by its three-term Maclaurin expansion, $1+\lambda t+\frac{1}{2} t^{2}\left(v+\lambda^{2}\right)$, where $v=\operatorname{var}\left(Z_{1}\right)$, whence the approximation $\alpha \approx-2 \lambda / v$ if $\lambda \approx 0$.

If $\varepsilon$ has the standard exponential law then Gabaix's (1999, p. 74950) account can be understood as taking $S_{\infty}=s_{\min } \mathrm{e}^{\varepsilon / a}$. A first consequence is that

$$
\mathrm{P}\left(S_{\infty}>x\right)=\mathrm{P}\left(\varepsilon a \log \left(\frac{x}{s_{\min }}\right)\right)=\left(\frac{x}{s_{\min }}\right)^{-a},
$$

i.e. that $a$ as defined above $i s$ the Zipf exponent for the RBM model. A second consequence is that

$$
\bar{s}=\frac{s_{\min }}{1-a^{-1}},
$$

provided that $a>1$. In this case, $a=\left(1-s_{\min } / \bar{s}\right)^{-1}$.

This reconstruction of Gabaix (1999) yields his identification of the Zipf exponent $a$ in terms of the limiting mean city size $\bar{s}$ and the base size $s_{\min }$. Thus, $a \approx 1$ if $\bar{s} \gg s_{\min }$. In terms of the RBM parameters, these conditions are that $\sigma^{2}-2 \mu$ is positive and close to 0 . Observed city 
sizes allow estimation of $\bar{s}$ and $a$, and these may be used to estimate $s_{\min }$. This could provide one check on the adequacy of Gabaix's model.

The heavy-traffic approximation and the RBM evaluation of the Zipf exponent coincide if $\mu=-\lambda$ and $\sigma^{2}=v$, i.e. if $a=\alpha$; see Asmussen (2003, p. 287). However, an example shows that Gabaix's determination does not hold in general. Denote the distribution function of the $Z_{n} \mathrm{~s}$, the increments of the underlying random walk, by $J(y)$. Let $\mu>0$ and $0<$ $\beta<1$, and suppose that $J(y)=1-\beta \mathrm{e}^{-\mu y}$ if $y \geq 0$. The drift of the random walk equals $D=\int_{-\infty}^{0} y \mathrm{~d} J(y)+\beta / \mu$, and if $D<0$ then the limit $W_{\infty}$ exists and $\omega(y)=1-q \mathrm{e}^{-\alpha y}$ $(y \geq 0)$, where $\alpha$ is the unique solution in $(0, \mu)$ of the equation $1-c(s)=\beta \mu /(\mu-s)$, where $c(s)=\int_{-\infty}^{0} \mathrm{e}^{s y} \mathrm{~d} J(y)$, and $q=1-\alpha / \mu$. This gives the exact power law for $S_{\infty}, \bar{\sigma}(x)=$ $q\left(x / s_{\min }\right)^{-\alpha}$, and $\mathrm{P}\left(S_{\infty}=s_{\min }\right)=1-q$. In addition, if $\alpha>1$ then $\bar{s}=s_{\min }(1+q /(\alpha-1))$, and, hence,

$$
\left(1-\frac{s_{\min }}{\bar{s}}\right)^{-1}=1+\frac{\alpha-1}{q}>\alpha .
$$

The product sequence $\left\{S_{n}\right\}$ is clearly a Markov chain with a state space $\delta$ which should be chosen as $[0, \infty)$ if $F(0)>0$ and $(0, \infty)$ if $F(0)=0$. Denote the transition kernel of this product chain by

$$
K(A \mid z)=\mathrm{P}\left(S_{n+1} \in A \mid S_{n}=z\right)=F\left(\frac{A}{z}\right)
$$

where $A$ is a Borel subset of $\&, A / z:=\{x / z: x \in A\}$, and $F(\cdot)=\mathrm{P}(X \in \cdot)$. Gabaix (1999, p. 744) effectively asserted that if $\mathrm{E}[X]=1$ then the measure $\mu$ defined by

$$
\mu(A)=\int_{A} \frac{\mathrm{d} z}{z}
$$

is a stationary law for the product chain. Moreover, he asserted that this explains the occurrence of Zipf's law with its unit exponent. There are two problems with this account. The first is that $\mu$ is an infinite measure and the product chain does not have a stationary law. The second is that there appears to be a conflation of the exponent of the tail of a power law and that of its density function.

If $F(0)>0$ then clearly the state $\{0\}$ is absorbing and its hitting time is almost surely finite. If $F(0)=0$ then $\{0\}$ is never hit, and the classification theory for random walks implies that

$$
\mathrm{P}\left(\left\{\limsup _{n \rightarrow \infty} S_{n}=\infty\right\} \cup\left\{\liminf _{n \rightarrow \infty} S_{n}=0\right\}\right)=1 .
$$

We will see in Theorem 6.1, below, that Gabaix's assertion about $\mu$ can be rendered as asserting that the measure $\mu$ is invariant provided only that $F(0)=0$. But first we check the existence of desirable regularity properties. We use the notation $\mathrm{E}_{z}[\cdot]=\mathrm{E}\left[\cdot \mid S_{0}=z\right]$.

If $h$ is a (Borel) measurable function and bounded on $\&$ then $\mathrm{E}_{z}\left[h\left(S_{1}\right)\right]=\mathrm{E}[h(z X)]$ is finite. If $h$ is also continuous then so is $\mathrm{E}_{z}\left[h\left(S_{1}\right)\right]$, and, hence, the product chain is weak Feller (Meyn and Tweedie (1993, p. 128)). Say that the product chain is strong Feller if $\mathrm{E}_{z}\left[h\left(S_{1}\right)\right]$ is continuous whenever $h$ is bounded and measurable. Demonstrating this property needs the invariant measure result foreshadowed above, so we state and prove it here.

Theorem 6.1. Assume that $F$ is nondegenerate. The measure (6.1) is invariant for the product chain if and only if $F(0)=0$. 
Proof. We have to show that $\mu(A)=\int_{0}^{\infty} K(A \mid z) \mu(\mathrm{d} z)$ for all Borel $A \subset(0, \infty)$. Countable additivity properties of $F(\cdot)$ and integrals imply that it suffices to consider intervals $A=(a, b]$, where $0<a<b<\infty$. In this case, $K(A \mid z)=F(b / z)-F(a / z)$, and, hence, the integral term equals

$$
\mathcal{I}(A):=\int_{0}^{\infty} \frac{F(b / z)-F(a / z)}{z} \mathrm{~d} z=\int_{0}^{\infty} \frac{F(b v)-F(a v)}{v} \mathrm{~d} v,
$$

a Frullani integral. The detailed evaluation of Frullani integrals depends on specific properties of the integrand function; compare the treatments of Bingham et al. (1987, p. 35) and Jeffreys and Jeffreys (1962, p. 406). Since $F$ is a distribution function, we can argue as follows (as in Hardy (1901, Section 2)). If $\epsilon>0$ then the infinite integral $\mathcal{L}(A)$ is the limit as $\epsilon \rightarrow 0$ of the integral over $\left[\epsilon, \epsilon^{-1}\right]$ and changing variables in an obvious way (e.g. $u=b v$ ) yields the evaluation

$$
\begin{aligned}
I(A) & =\lim _{\epsilon \rightarrow 0}\left(\int_{a / \epsilon}^{b / \epsilon} \frac{F(u)}{u} \mathrm{~d} u-\int_{a \epsilon}^{b \epsilon} \frac{F(u)}{u} \mathrm{~d} u\right) \\
& =(F(\infty-)-F(0+)) \log \left(\frac{b}{a}\right) \\
& =(1-F(0)) \mu(A) .
\end{aligned}
$$

The assertion follows.

Our next result is very closely related to Proposition 6.1.3 of Meyn and Tweedie (1993). Note that the proof of Theorem 6.1 shows that $\mu$ is super-invariant for any $F$.

Theorem 6.2. The product Markov chain is strong Feller if and only if $F$ is absolutely continuous in $(0, \infty)$ with respect to the Lebesgue measure.

Proof. If $F$ has this property then

$$
\mathrm{E}_{z}\left[h\left(S_{1}\right)\right]=h(0) F(0)+\mathrm{E}\left[h\left(\mathrm{e}^{\log z+\log X} ; X>0\right)\right]=h(0) F(0)+\int h\left(\mathrm{e}^{u}\right) g(u-\log z) \mathrm{d} u,
$$

where $g(u)=\mathrm{e}^{u} F^{\prime}\left(\mathrm{e}^{u}\right)$ is the density of the law of $Z$ restricted to the real line. So if $\delta$ is real then

$$
\left|\mathrm{E}_{z+\delta}\left[h\left(S_{1}\right)\right]-\mathrm{E}_{z}\left[h\left(S_{1}\right)\right]\right| \leq\left[\sup _{z \geq 0}|h(z)|\right] \int|g(u-\log (z+\delta))-g(u-\log z)| \mathrm{d} u,
$$

and the integral term tends to 0 uniformly with respect to $z \in \mathcal{R}=(-\infty, \infty)$ as $\delta \rightarrow 0$, as shown in Theorem 9.5 of Rudin (1974, p. 196). Thus, $\left\{S_{n}\right\}$ is strong Feller.

To prove the converse, note that the strong Feller property is equivalent to the lower semicontinuity for all Borel $A$ of $K(A \mid z)$ (Meyn and Tweedie (1993, p. 128). Choose $A \subset(0, \infty)$ such that $\xi=F(A)>0$. Lower semicontinuity implies that there is an open neighbourhood of unity, denoted by $I$, such that $K(A \mid z) \geq \frac{1}{2} K(A \mid 1)=\xi / 2$ for all $x \in I$. Since the measure (6.1) is super-invariant, it follows that

$$
\infty>\mu(A) \geq \int_{0}^{\infty} K(A \mid z) \mu(\mathrm{d} z) \geq \int_{I} K(A \mid z) \mu(\mathrm{d} z) \geq \frac{1}{2} \xi \mu(I)>0 .
$$

This inequality implies that $F$ is absolutely continuous in $(0, \infty)$ with respect to $\mu$, and the converse assertion follows. 
The question we now address is that of uniqueness of invariant measures. If we accept that $0 \in \delta$ then it is clear that the point mass at $\{0\}$ is invariant. We will ignore this contingency, and restrict the search to nontrivial measures. We need to distinguish the cases where the underlying random walk is arithmetic and nonarithmetic. In the former case we can, with no loss of generality, assume that there is a number $Q>1$ such that $\delta=\left\{Q^{i}: i=0, \pm 1, \ldots\right\}$ is the essential range of $X$. So if $S_{0} \in \delta$ then $S_{n} \in \delta$ for all $n$. Let $f_{i}=\mathrm{P}\left(X=Q^{i}\right)$, and assume that the discrete law assigning mass $f_{i}$ to $i$ has unit maximal span. Thus, the product chain becomes a discrete-state Markov chain with one-step transition probability

$$
p_{i j}=\mathrm{P}\left(S_{n+1}=Q^{j} \mid S_{n}=Q^{i}\right)=\mathrm{P}\left(X=Q^{j-i}\right)=f_{j-i} .
$$

Since the state space is discrete, it makes sense to restrict attention to discrete invariant measures.

The right-hand side of (6.2) is the transition probability for a random walk on the integers. It is known that the counting measure is invariant for any such random walk, so it follows that assigning a constant mass to states $Q^{i}$ gives an invariant measure for the product chain. The maximal span assumption ensures that the random walk is irreducible, so if it is recurrent then this invariant measure is unique (i.e. up to a constant multiplier, which convention will be understood in the sequel). This is the case if $\lambda=0$.

If the random walk is transient then it can be the case that there exists a positive number $\rho \neq 1$ such that

$$
\sum_{i} f_{i} \rho^{i}=1
$$

A necessary condition is that $f_{|i|}$ decreases faster than some geometric sequence. If (6.3) is satisfied then

$$
\sum_{i} \rho^{-i} p_{i j}=\rho^{-j} \sum_{i} \rho^{j-i} f_{j-i}=\rho^{-j}
$$

Hence, allocating mass $\rho^{-i}$ to $Q^{i}$ yields another invariant measure, and convex combinations of these two measures are invariant. It follows from a result used to prove Theorem 6.3, below, that these are the only possibilities and, hence, that the counting measure is unique if (6.3) has no solution.

Now assume that the underlying random walk is nonarithmetic. If it is recurrent then intervals are recurrent sets (i.e. almost surely, the random walk hits every interval infinitely often). It follows that open sets are recurrent. The same is obviously true for the product chain, and since it has an invariant measure, it follows that it is Harris recurrent (i.e. $\mu(A)>0$ implies that $A$ is recurrent). It further follows that $\mu$ specified by (6.1) is the unique super-invariant measure (Revuz and Yor (1991, p. 395)).

There seems to be almost no general theory about invariant measures for transient Markov chains. Examples show that invariant measures may not exist, exist uniquely, or exist in abundance. The following result resolves our case in some generality, and it reveals the sense in which power-tail functions are associated with the product chain.

Theorem 6.3. Suppose that $F(0)=0$ and that 8 is the smallest multiplicative group containing $\operatorname{supp}(F)$. If $\mu$ is invariant and assigns finite measure to compact subsets of 8 , then $\mu(\mathrm{d} x)=$ $\phi(x) \mathrm{d} x$, where

$$
\phi(x)=\int_{\mathcal{A}(F)} x^{-1-a} \xi(\mathrm{d} a)
$$

and $\xi$ is a measure supported in

$$
\mathcal{A}(F):=\{a \in \mathcal{R}: m(a)=1\} .
$$


Remarks. (i) The set $\mathcal{A}(F)$ contains $\{0\}$ and it may contain a distinct second element $\{\alpha\}$ such that $m(\alpha)=1$. If this holds then the most general invariant measure is

$$
\mu(\mathrm{d} x)=\left(C_{1} x^{-1}+C_{2} x^{-1-\alpha}\right) \mathrm{d} x,
$$

where $C_{1}, C_{2} \geq 0$ and $C_{1}+C_{2}>0$.

(ii) The assumption of Theorem 6.3 is met if $\operatorname{supp}(F)$ contains an interval.

Proof of Theorem 6.3. If $A$ is a Borel subset of $\&$ then

$$
\mu(A)=\int_{\S} \int_{A / x} F(\mathrm{~d} v) \mu(\mathrm{d} x)=\int_{\S} \int_{A / v} \mu(\mathrm{d} x) F(\mathrm{~d} v),
$$

since $v \in A / x$ if and only if $x \in A / v$. Define the measure $v$ on $\mathcal{R}$ by $v(B)=\mu\left(\mathrm{e}^{-B}\right)$, where $\mathrm{e}^{-B}=\left\{x: x=\mathrm{e}^{-y}, y \in B\right\}$ with $B$ a Borel subset of $\mathcal{R}$. Similarly, let $\sigma(B)=F\left(\mathrm{e}^{B}\right)$, so if $v=\mathrm{e}^{y}$ then $\sigma(\mathrm{d} y)=F(\mathrm{~d} v)$. The inner integral on the right-hand side of (6.6) equals

$$
\int_{\S} \mathbf{1}_{\exp (-(y+B))}(x) \mu(\mathrm{d} x)=v(y+B) .
$$

Thus, (6.6) takes the form

$$
v(B)=\int_{\mathcal{R}} v(y+B) \sigma(\mathrm{d} y)
$$

an integrated Cauchy functional equation.

The assumption relating $\&$ and $\operatorname{supp}(F)$ is equivalent to $\mathcal{R}$ being the smallest closed additive subgroup containing $\operatorname{supp}(\sigma)$. It follows from Theorem 9.5.3 of Ramachandran and Lau (1991, p. 231) that $v(\mathrm{~d} y)=h(y) \mathrm{d} y$, where $h(y)=\int_{\mathcal{A}(F)} \mathrm{e}^{a y} \theta(\mathrm{d} a)$ and $\theta$ is a measure supported in $\mathcal{A}(F)$; note that $\mathcal{A}(F)$ defined in (6.5) coincides with the set $\left\{a \in \mathcal{R}: \int_{\mathcal{R}} \mathrm{e}^{a y} \sigma(\mathrm{d} y)=1\right\}$. Thus, the density of $v$ (with respect to the Lebesgue measure) is a mixture of exponential functions $\mathrm{e}^{a y}$. It follows that $\mu$ is absolutely continuous and that its density is obtained from the inverse map $y=-\log x$ as

$$
\left|\frac{\mathrm{d} y}{\mathrm{~d} x}\right| \mathrm{e}^{a(-\log x)}=x^{-1-a},
$$

and (6.4) follows.

Conversely, if $\alpha \neq 0$ exists then taking $A=(a, b]$ in (6.6) it is easy to show that $\mu(\mathrm{d} x)=$ $x^{-1-\alpha} \mathrm{d} x$ is invariant. This completes the proof.

An interpretation of invariant measures as a statistical equilibrium of an infinite system of noninteracting particles is due to Derman (1955, Theorem 2) in the case of Markov chains having a countable state space. An extension to more general cases is described briefly in Daley and Vere-Jones (1988, pp. 421-2). Applied to the product chain, identify cities with their sizes as points in $\delta=(0, \infty)$. Assume that at time $n=0$ there are infinitely many cities whose sizes comprise a Poisson $(\mu)$ random field. This means that there is a random measure $N_{0}(\cdot)$ defined on Borel subsets $A$ of $\&$ such that $N_{0}(A) \sim \operatorname{Po}(\mu(A))$, and if $A_{1}, \ldots, A_{k}(k=2,3, \ldots)$ are disjoint then $N_{0}\left(A_{1}\right), \ldots, N_{0}\left(A_{k}\right)$ are independent. Assume further that the sizes of a given city at times $n=1,2, \ldots$ evolve according to the product chain, and that the evolutions of differing cities are independent. If $\mu$ is the invariant measure in Theorem 6.3 and if $N_{n}(A)$ is the number of cities at time $n$ with sizes in $A$, then $N_{n}(\cdot)$ is a Poisson $(\mu)$ random field. 
Now suppose that $\lambda<0$. So if $\mathcal{A}(F)$ defined in (6.5) contains two elements then the second one is $\alpha>0$. If $b>0$ is fixed and $A=(x, x+b]$, then it follows from Theorem 6.3 that

$$
\begin{aligned}
\mathrm{E}\left[N_{n}(A)\right] & =C_{1} \log \left(1+\frac{b}{x}\right)+\left(\frac{C_{2}}{\alpha}\right) x^{-\alpha}\left(1-\left(1+\frac{b}{x}\right)^{-\alpha}\right) \\
& =\frac{b}{x}\left(C_{1}+C_{2} x^{-\alpha}\right)\left(1+o\left(x^{-1}\right)\right), \quad x \rightarrow \infty .
\end{aligned}
$$

This is a sense in which Zipf's law holds with unit exponent independently of the form of $F$.

\section{Acknowledgement}

I thank the anonymous referee for the close attention given to earlier versions of this paper.

\section{References}

Adler, R. J., Feldman, R. E. And Taqqu, S. (eds) (1998). A Practical Guide to Heavy Tails. Birkhauser, Boston, MA. Albin, J. M. P. (2008). A note on the closure of convolution power mixtures (random sums) of exponential distributions. J. Austral. Math. Soc. 84, 1-7.

Asmussen, S. (2003). Applied Probability and Queues, 2nd edn. Springer, New York.

Bingham, N. H., Goldie, C. M. And Teugels, J. L. (1987). Regular Variation. Cambridge University Press.

Cline, D. B. H. (1987). Convolutions of distributions with exponential and subexponential tails. J. Austral. Math. Soc. Ser. A 43, 347-356. (Correction: 48, (1990), 152-153.)

Daley, D. J. AND Vere-Jones, D. (1988). An Introduction to the Theory of Point Processes. Springer, New York.

Derman, C. (1955). Some contributions to the theory of denumerable Markov chains. Trans. Amer. Math. Soc. 79, $541-555$.

Feller, W. (1971). An Introduction to Probability Theory and Its Applications, Vol. 2, 2nd edn. John Wiley, New York. Foss, S. And Korshunov, D. (2007). Lower limits and equivalences for convolution tails. Ann. Prob. 35, 366-383.

GabaiX, X. (1999). Zipf's law for cities: an explanation. Quart. J. Econom. 114, 739-767.

Gnedenko, B. V. And Korolev, V. Y. (1996). Random Summation: Limit Theorems and Applications. CRC Press, Boca Raton, FL.

HaRdy, G. H. (1901). On the Frullanian integral $\int_{0}^{\infty}\left(\phi\left(a x^{m}\right)-\phi\left(b x^{m}\right)\right)(\log x)^{p} x^{-1} d x$. Quart. J. Math. 33, $113-144$.

Harrison, J. M. (1985). Brownian Motion and Stochastic Flow Systems. John Wiley, New York.

HaYes, B. (2007a). Fat tails. Amer. Scientist 95, 200-204.

HAYES, B. (2007b). Web log. http://bit-player.org.

Jefrreys, H. And Jeffreys, B. S. (1962). Methods of Mathematical Physics. Cambridge University Press.

Kalashnikov, V. V. (1997). Geometric Sums: Bounds for Rare Events with Applications. Kluwer, Dordrecht.

Korshunov, D. (1997). On the distribution tail of the maximum of a random walk. Stoch. Processes Appl. 72, 97-103. Meyn, S. AND Tweedie, R. L. (1993). Markov Chains and Stochastic Stability. Springer, London.

MitzenmaCher, M. (2004). A brief history of generative models for power law and lognormal distributions. Internet Math. 1, 226-251.

Newman, M. E. J. (2005). Power laws, Pareto distributions and Zipf's law. Contemp. Phys. 46, 323-351.

PaKes, A. G. (2004). Convolution equivalence and infinite divisibility. J. Appl. Prob. 41, 407-424.

Pakes, A. G. (2007). Convolution equivalence and infinite divisibility: corrections and corollaries. J. Appl. Prob. 44, 294-304.

RaCHEv. S. (ed.) (2003). Handbook of Heavy Tailed Distributions in Finance. Elsevier, Amsterdam.

Ramachandran, B. And Lau, K. S. (1991). Functional Equations in Probability Theory. Academic Press, San Diego.

ReEd, W. J. AND Hughes, B. D. (2002). From gene families and genera to incomes and internet file sizes: Why power laws are so common in nature. Phys. Rev. E 66, 067103, 4 pp.

Revuz, D. AND Yor, M. (1991). Continuous Martingales and Brownian Motion. Springer, Berlin.

Rudin, W. (1974). Real and Complex Analysis, 2nd. edn. McGraw-Hill, New York.

Watanabe, T. (2008). Convolution equivalence and distributions of random sums. Prob. Theory Relat. Fields 142, 367-397. 